\title{
Recent development of SPR spectroscopy as potential method for diagnosis of dengue virus E-protein
}

\begin{abstract}
Purpose: This paper aims to review the potential application of surface plasmon resonance (SPR) in diagnosis of dengue virus (DENV-2) E-protein and the development of SPR to become an alternative DENV sensor. Design/methodology/approach: In this review, the existing standard laboratory techniques to diagnosis of DENV are discussed, together with their drawbacks. To overcome these drawbacks, SPR has been aimed to be a valuable optical biosensor for identification of antibodies to the DENV antigen. The review also includes the future studies on three-dimensional poly(amidoamine) (PAMAM) dendrimer-surfaceassembled monolayer (SAM)-Au multilayer thin films, which are envisaged to have high potential sensitive and selective detection ability toward target E-proteins. Findings: Application of SPR in diagnosis of DENV emerged over recent years. A wide range of immobilized biorecognition molecules have been developed to combine with SPR as an effective sensor. The detection limit, sensitivity and selectivity of SPR sensing in DENV have been enhanced from time to time, until the present. Originality/value: The main purpose of this review is to provide authors with up-to-date and useful information on sensing DENV using SPR and to introduce a novel three-dimensional PAMAM-SAM-Au multilayer thin films for future research on SPR sensing applications.
\end{abstract}

Keyword: DENV E-protein; IgM antibody; PAMAM dendrimer; Surface plasmon resonance 